\title{
Anomalous Pulsar Scattering at LOFAR Frequencies
}

\author{
Marisa Geyer ${ }^{1}$ and Aris Karastergiou ${ }^{1,2,3}$ \\ ${ }^{1}$ Astrophysics, University of Oxford, \\ Denys Wilkinson Building, Keble Road, Oxford OX1 3RH, UK \\ email: marisa.geyer@physics .ox.ac.uk \\ ${ }^{2}$ Physics Department, University of the Western Cape, \\ Cape Town 7535, South Africa \\ ${ }^{3}$ Department of Physics and Electronics, Rhodes University, \\ PO Box 94, Grahamstown 6140, South Africa
}

\begin{abstract}
The Low Frequency Array (LOFAR) is ideally suited to pulsar scattering studies, providing broad bands at low frequencies where the imprints of the ionized Interstellar Medium (IISM) are exaggerated. We analyse a set of sources at 110-190 MHz, and find unexpectedly shallow dependencies of pulse scatter broadening on frequency. These anomalous scattering values are discussed by considering evidence for anisotropic scattering and small scattering clouds.
\end{abstract}

Keywords. pulsars: general, scattering, ISM: structure

\section{Introduction}

Fifty years after the discovery of the CP1919 at $\sim 80 \mathrm{MHz}$, pulsar astronomy is experiencing a "low-frequency pulsar renaissance" (see Hessels et al. in these proceedings). Low frequency pulsar science provides an opportunity to investigate the detailed characteristics of the IISM. This includes studies of the variability and dependencies of pulsar dispersion measures (DMs) and pulsar scattering measurements (Pilia et al. 2016, Bilous et al. 2016). Here, we analyse the scatter broadening of 13 LOFAR pulsars at High Band Antenna (HBA) frequencies $(110-190 \mathrm{MHz})$. We find anomalous scattering spectral indices, which could suggest the presence of anisotropic scattering mechanisms and/or finite scattering clouds in the IISM.

\section{Pulsar Scattering Overview}

Pulsar scattering is caused by fluctuations in the electron densities of the IISM. These fluctuations lead to the multipath propagation of radio waves, observed as scattering tails in average pulse profiles. To model these effects, we describe a scattering angle distribution associated with the IISM, and its frequency dependence. A typical framework within which scattering models are constructed is the thin screen approximation (Williamson 1972). The thin screen model assumes that the radio wave scattering takes place at a single location along the line of sight (Fig. 1). The screen is modelled to be infinitely large transverse to the direction of the radio wave propagation.

We consider two scattering mechanisms: isotropic and anisotropic. An isotropic screen scatters radio waves equally in all directions, as e.g. a circularly symmetric Gaussian distribution in scattering angle $a$. This distribution leads to a temporal broadening function $(\mathrm{BF}) \sim e^{-t / \tau}$, where $\tau$ is the characteristic scattering delay with a frequency dependence 


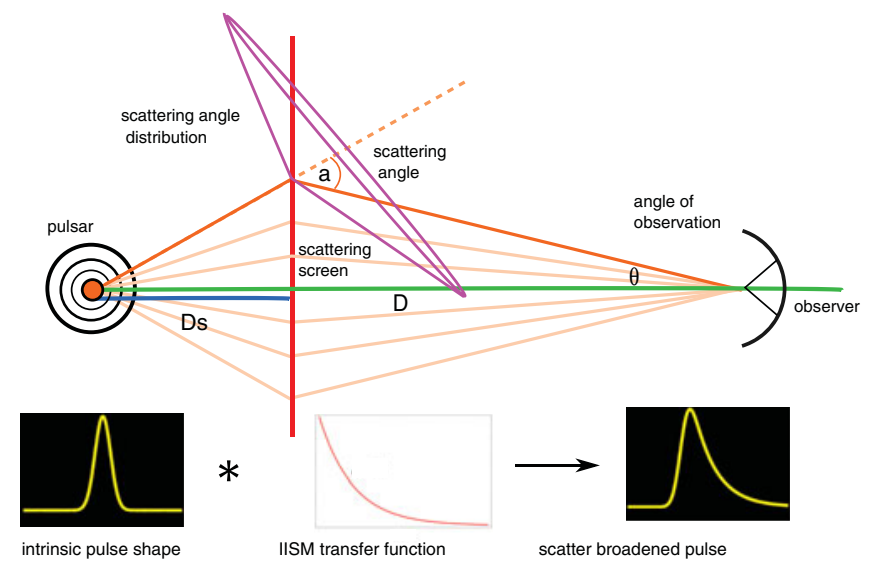

Figure 1. A schematic of the thin screen scattering setup, with the pulsar at an overall distance $D$ and the screen at a distance $D_{s}$ from the pulsar. Simple geometric relationships exist between the small scattering angle $(a)$ and the angle $(\theta)$ at which the scattered ray is observed, as well as the time delay corresponding to the extra path length travelled (see e.g. Williamson 1972).

$\tau \propto \nu^{-\alpha}$ and $\alpha=4$. An anisotropic scattering mechanism exhibits directionality in its distribution of scattering angles, i.e. the screen scatters more strongly in one direction than another. We use an asymmetric Gaussian distribution as an example of this, leading to a more complex temporal BF with two scattering timescales, each of which $\tau_{x, y} \propto \nu^{-4}$ (Geyer et al. 2016). In the limit where the screen scatters along one dimension only (e.g. $\left.\tau_{x} \gg \tau_{y}\right)$, the BF is given by $e^{-t / \tau} /(\sqrt{\pi t \tau})$. Deviations from these theoretical dependencies have both been proposed (e.g. Cordes \& Lazio 2001) and observed. These include $\alpha$ values smaller than the predicted values, as in Löhmer et al. (2001), and recently in Lewandowski et al. (2015), hereafter L15.

\section{Fitting Techniques}

The fitting techniques used to extract scattering values and intrinsic profile parameters are described in Geyer et al. (2016). Here we highlight a single feature of the fitting technique, namely to introduce a DC offset parameter in the presence of high levels of IISM scattering. Severe levels of scattering leads to average profile shapes with scattering tails that wrap around the full rotational pulse phase, and will raise the observing baseline (compared to the unscattered pulse). By including a DC fitting parameter, we ensure that the obtained $\tau$ 's are accurate, while providing an estimate of the flux lost to the raised baseline. The fitting method also provides small DM corrections $(\Delta \mathrm{DM})$ due to scattering. Typically, in pulsar observations the best DM value is the DM value that maximizes the signal-to-noise ratio (SNR) of the average profile. However, if the profile is scattered across the observing band, the DM value that optimizes the scattered profile's SNR will be an overestimation of the DM that would align the intrinsic unscattered profiles.

\section{LOFAR Data Analysis}

The results for 13 scattered LOFAR pulsars are presented in Table 1. A description of the data and the detailed analyses is in Geyer et al. (2017). We present example results for two sources in Fig. 2. The left plots of each shows a single profile shape fitted with both the isotropic model (IM; solid) and the extreme anisotropic model ( $\mathrm{AM}_{1 \mathrm{D}}$; dashed). The middle plots show the $\tau$ spectra. For both models, the $\alpha$ values are significantly lower 
Table 1. List of obtained $\tau$ values at $150 \mathrm{MHz}$, and spectral indices, $\alpha$, using two models.

\begin{tabular}{lcc|ccc}
\hline Pulsar & IM: $\alpha$ & $\mathrm{AM}_{1 \mathrm{D}}: \alpha$ & Pulsar & IM: $\alpha$ & $\mathrm{AM}_{1 \mathrm{D}}: \alpha$ \\
\hline J0040+5716 & $2.2 \pm 0.2$ & $2.7 \pm 0.3$ & $\mathrm{~J} 1913-0440$ & $3.3 \pm 0.1$ & $4.1 \pm 0.2$ \\
$\mathrm{~J} 0117+5914$ & $1.9 \pm 0.2$ & $2.6 \pm 0.2$ & $\mathrm{~J} 1917+1353$ & $2.8 \pm 0.4$ & $3.6 \pm 0.6$ \\
$\mathrm{~J} 0543+2329$ & $2.6 \pm 0.2$ & $2.7 \pm 0.3$ & $\mathrm{~J} 1922+2110$ & $2.0 \pm 0.2$ & $3.3 \pm 0.4$ \\
$\mathrm{~J} 0614+2229$ & $2.1 \pm 0.1$ & $3.1 \pm 0.3$ & $\mathrm{~J} 1935+1616$ & $3.4 \pm 0.2$ & $3.9 \pm 0.5$ \\
J0742-2822 & $3.8 \pm 0.4$ & $\ldots$ & $\mathrm{J} 2257+5909$ & $2.6 \pm 0.4$ & $3.4 \pm 0.6$ \\
$\mathrm{~J} 1851+1259$ & $4.1 \pm 0.3$ & $4.7 \pm 0.4$ & $\mathrm{~J} 2305+3100$ & $1.5 \pm 0.1$ & $2.0 \pm 0.1$ \\
J1909+1102 & $3.5 \pm 0.4$ & $6.4 \pm 0.7$ & & & \\
\hline$\langle\alpha\rangle$ & $2.7 \pm 0.2$ & $3.5 \pm 0.4$ & &
\end{tabular}
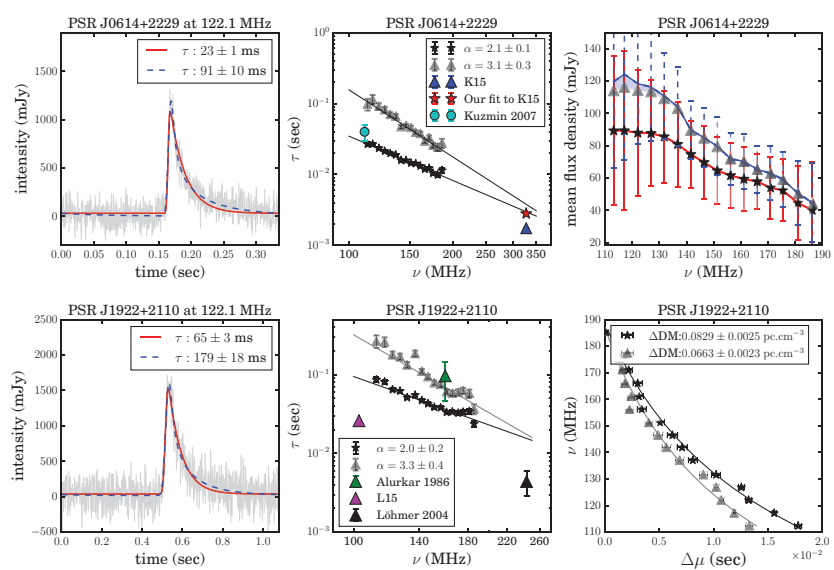

Figure 2. Scattering analyses of PSRs J0614+2229 \& J1922+2110. Details in the text.

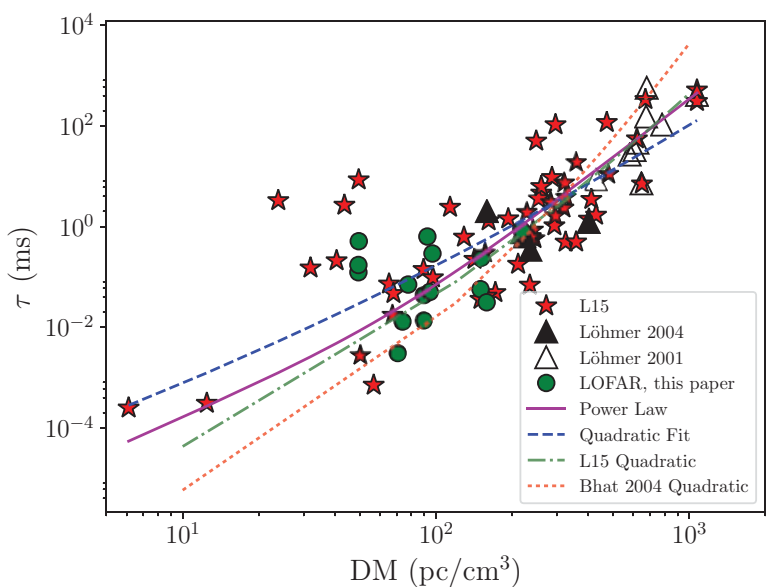

Figure 3. The $\tau$ values at $1 \mathrm{GHz}$ plotted against their corresponding DM values. The datapoints include values from the literature as shown in the caption. The combined best power law fit (solid) and quadratic fit (dashed) are compared to the trends obtained by L15 (dot-dash) and Bhat et al. (2004; dotted).

than theoretical predictions. The $\alpha$ values using the $\mathrm{AM}_{1 \mathrm{D}}$ are larger than for the IM. The flux spectrum of PSR J0614+2229 is shown in the top right. The shaded region represents flux recovered by the fitting model, due to a wrap-around scattering tail. In the bottom right we show the DM corrections ( $\triangle \mathrm{DM}$, as described in Sec. 3) for PSR J1922+2110. For all spectra, the IM is in stars and the $\mathrm{AM}_{1 \mathrm{D}}$ in triangles. The overview presented 
in Table 1 , shows that $\langle\alpha\rangle$ is smaller than 4 for both the IM and $\mathrm{AM}_{1 \mathrm{D}}$, but closer to a theoretical value of 4 for the $\mathrm{AM}_{1 \mathrm{D}}$.

\section{Discussion and Outlook}

Discussion What is the origin of these anomalously low $\alpha$ values at low frequencies? One possibility is the presence of small scattering clouds, different from the transversely extended thin scattering screen of Fig 1. In Geyer et al. (2017) we show that the $\alpha$ distribution of Table 1 can be obtained through midway truncated scattering screens of sizes $\sim 100 \mathrm{AU}$. Another possibility is through anisotropic scattering mechanisms. Analysis of secondary pulsar spectra by e.g. Stinebring et al. (2001), and observations by Brisken et al. (2010) have already provided evidence for anisotropy within the IISM. Anisotropic modelling (using $\mathrm{AM}_{1 \mathrm{D}}$ ), is not strictly required to fit the LOFAR data shown here. However, as described in Geyer et al. (2017), potential evidence for anisotropy is found for a small set of sources. Anisotropic scattering mechanisms are certainly methods by which to create apparent low $\alpha$ values. Using simulations, we show in Geyer et al. (2016) that fitting anisotropically scattered profiles naievly with isotropic models can lead to low $\alpha$ values, similar to those obtained in Table 1.

Fig. 3 considers how the LOFAR results compare to the established Bhat et al. (2004) trend, which suggests a quadratic dependence of $\tau$ at $1 \mathrm{GHz}$ on DM on a log-log scale. Values obtained by L15 and our own promote higher $\tau$ values at especially low DM values, with a large spread in values. For the Bhat et al. (2004) relationship to hold at $1 \mathrm{GHz}$, we would require a frequency dependent $\alpha$ value.

Outlook While a time domain analysis alone is not the most sensitive for analyzing IISM properties, we do find anomalous scattering effects using single epoch average pulse profiles. Higher SNR profiles at low frequencies will allow us to distinguish between scattered profile shapes (IM vs AM). Added high resolution dynamic spectra will aid tests for anisotropy (see Stinebring et al. in these proceedings). Furthermore, broad band scintillation results will provide precise scattering measurements at higher frequencies to test the frequency dependence of $\alpha$. Lastly, interferometric imaging, including spaceground experiments, could be key in investigating the typical sizes of scattering surfaces.

\section{References}

Bilous, A. V., Kondratiev, V. I., Kramer, M. et al. 2016, A\&SA, 591, A134

Pilia, M., Hessels, J. W. T., Stappers, B. W. et al. 2016, A\&A, 586, A92

Williamson, I. P., 1972 MNRAS, 157, 55

Geyer, M. \& Karastergiou, A., 2016 MNRAS, 462, 2587

Geyer, M., Karastergiou, A., Kondratiev, V. I., et al., 2017 MNRAS, 470, 2659

Brisken, W. F., Macquart, J.-P., Gao, J. J., et al., 2010 ApJ, 708, 232

Stinebring, D. R., McLaughlin, M. A., Cordes, J. M., et al., 2001 ApJL, 549, L97

Cordes, J.M., Lazio, T.J.W., 2001 ApJ, 549, 997

Bhat, N. D. R., Cordes, J. M., Camilo, F., Nice, D. J., and Lorimer, D. R., 2004 ApJ, 605, 759

Löhmer, O., Kramer, M., Mitra, D., Lorimer, D. R., \& Lyne, A. G., 2001 ApJL, 562, L157

Lewandowski, W., Kowalińska, M., \& Kijak, J., 2015 MNRAS, 449, 1570 